This is an open access article distributed under the terms of the Creative Commons BY-NC-ND Licence

\title{
The gene $O S I A A 9$ encoding auxin/indole-3-acetic acid proteins is a negative regulator of auxin-regulated root growth in rice
}

\author{
Y.L. SONG* \\ Anhui Key Laboratory for Biodiversity Research and Ecological Conservation on Southwest Anhui, College of Life \\ Science, Anqing Normal University, Anqing, 246011, P.R. China
}

\begin{abstract}
Auxin signal transduction in plants depends on regulation by short-lived nuclear plant proteins called auxin/indole-3acetic acid (Aux/IAA) proteins. The OsIAA9, which is highly and rapidly induced by auxin, encodes the entire Aux/IAA domain characteristic of the Aux/IAA family in rice. The promoter region of OSIAA9 contains several cis-elements. Analysis of $\mathrm{P}_{\text {OsIAA9: }}$ :GUS transgenic plants indicates a specific expression of OsIAA9 in roots and seedling shoots, especially lateral roots and root tips. Based on real-time PCR, the expression of OSIAA9 was induced by multiple hormones and abiotic stresses. Furthermore, ectopic overexpression of OSIAA9 in rice led to fewer crown and lateral roots and reduced the inhibition of root elongation by auxin. These observations indicate that $O S I A A 9$ was a negative regulator of auxin-regulated root growth.
\end{abstract}

Additional key words: abscisic acid, crown and lateral roots, gibberelic acid, jasmonates, kinetin, Oryza sativa, salicylic acid, transgenic plants.

\section{Introduction}

Auxin, the first plant hormone discovered, regulates multiple plant developmental processes including, but not limited to, apical dominance, lateral root formation, vascular differentiation, embryo patterning, and shoot elongation (Muday and DeLong 2001, Jenik and Barton 2005, Leyser 2005). Auxin/indole-3-acetic acid (Aux/IAA) proteins are plant-specific transcriptional regulators, which share a four-domain structure. They act as co-receptors with auxin receptor transport inhibitor response 1 (TIR1) and function as transcriptional repressors by interacting with auxin response factor (ARF) proteins through conserved domains III and IV. Rice has 31 potential non-redundant Aux/IAA transcription factor genes (Jain et al. 2006, Song et al. 2009a), and the function of some has been identified. Some of them function in root development, such as OSIAA3 in crown root formation, OsIAA11 and OSIAA13 in lateral root development (Kitomi et al. 2012, Zhu et al. 2012), and OsIAA23 in postembryonic maintenance of the quiescent center in

roots (Ni et al. 2011). Understanding the regulation of root architecture in crop plants is of great agronomic importance because root architecture is a key determinant of plant anchorage and efficiency of water and nutrient uptake and adjusts plant growth to handle abiotic stresses. Unlike Arabidopsis, which has a primary root that iteratively branches to generate several orders of lateral roots, rice has predominantly shoot-borne crown roots that branch sequentially to form a herringbone-like structure (Rebouillat et al. 2009). Thus, unravelling genes which are involved in root development is key for improving agronomic characteristics of rice. We previously found that OSIAA9 and OsIAA20 are sister genes, which are highly and rapidly induced by auxin. They are tissue- or organ-specific, and they are up-regulated in plumules grown in the dark (Song et al. 2009a). Constitutive expression of OsIAA9 in Arabidopsis leads to a reduced gravitropic response and alters the number and distribution of starch granules (Luo et al. 2015).

Submitted 12 July 2017, last revision 27 July 2018, accepted 1 August 2018.

Abbreviations: ABA - abscisic acid; Aux/IAA - auxin/indole-3-acetic acid; ARF - auxin response factor; 2,4-D - 2,4-dichlorophenoxyacetic acid; GUS - $\beta$-glucuronidase; IAA - indole-3-acetic acid; JA - jasmonic acid; MS - Murashige and Skoog; SA - salicylic acid; TIR1 - transport inhibitor response 1.

Acknowledgements: This work was supported by grants from the Anhui Provincial Natural Science Foundation (1608085QC53) and the Anhui Provinicial Excellent Top Talent in Colleges and Universities Program (gxgwfx2018050).

*E-mail: songyaling82@163.com 
The aim of this research was to identify the function and localization of OSIAA9 in rice and to follow OSIAA9 expression induced by multiple hormones and abiotic

\section{Materials and methods}

Constructs and rice transformation: To make an overexpression construct, the full-length cDNA of OSIAA9 was amplified from Minghui 63 (Oryza sativa L. ssp. indica) by reverse transcription (RT)-PCR using primers 5'-ATAGGTACCAGAAATCCAGCC AAGAAG-3' and 5'-TATGGATCCTGAGATGTG CCCAACTACA-3'. The sequencing-confirmed PCR fragment was digested by $K p n \mathrm{I}$ and BamHI and inserted into pCAMBIA1301U. A $1.7 \mathrm{~kb}$ fragment containing the OSIAA9 promoter region was amplified from genomic DNA of an upland rice (Oryza sativa L. ssp. japonica) cv. IRAT109 with primers 5'-TATAAGCTTAAT CTTTGGTTCCGAGTTA-3' and 5'-TATGGATCCCTT CTTGGCTGGATTTCTA-3', and the sequencing-confirmed PCR fragment was digested by HindIII and BamHI and placed into pCAMBIA1391ZGUS (www.cambia.org, AF234312). Both constructs were transformed into japonica rice cv. Zhonghua11, a genotype supporting a highly efficient transformation using the Agrobacterium-mediated transformation method. Transgenic calli and plantlets were selected based on hygromycin $\left(50 \mathrm{mg} \mathrm{dm}^{-3}\right)$ resistance.

Plants and treatments: Zhonghual1 seedlings were germinated and grown at a temperature of $28^{\circ} \mathrm{C}$, a 14-h photoperiod, an irradiance of $50 \mu \mathrm{mol} \mathrm{m} \mathrm{m}^{-2} \mathrm{~s}^{-1}$, and an air humidity of $60 \%$ for about three weeks before treatment with phytohormones. The phytohormones used were: $100 \mu \mathrm{M}$ indole-3-acetic acid (IAA), $100 \mu \mathrm{M}$ 2,4-dichlorophenoxyacetic acid (2,4-D), $100 \mu \mathrm{M}$ jasmonic acid (JA), $100 \mu \mathrm{M}$ salicylic acid (SA), $100 \mu \mathrm{M}$ abscisic acid (ABA), $100 \mu \mathrm{M}$ gibberellic acid (GA), and $100 \mu \mathrm{M}$ kinetin (KT). Treatments lasted for 6 or $12 \mathrm{~h}$. Detailed information on drought (Dro) and salinity (Salt) treatments is in Song et al. (2009a)

Transgenic seeds were germinated on a Murashige and Skoog (MS) medium. Plantlets were transferred onto an MS medium containing different concentration of hormones and grown at $28^{\circ} \mathrm{C}$, a 14-h photoperiod, and an irradiance of $50 \mu \mathrm{mol} \mathrm{m} \mathrm{m}^{-2} \mathrm{~s}^{-1}$. Detailed information on plant treatments is in Song et al. (2009a). Methods used for auxin treatment of transgenic plants are described in Song et al. (2009b), and Song and Xu 2013).

Blotting RNA and real-time PCR: Total RNA was isolated from $\mathrm{T} 0$ rice leaves using a TRIzol reagent stresses. We also used OsIAA9-overexpressing plants, which had fewer crown roots, were defective in lateral root formation, and were resistant to exogenous auxin.

(Invitrogen, Carlsbad, CA, USA). The total RNA $(15 \mu \mathrm{g})$ from 24 samples was resolved on a $1.2 \%(\mathrm{~m} / \mathrm{v})$ agarose gel containing $2 \%(\mathrm{~m} / \mathrm{v})$ formaldehyde and blotted onto Hybond (Invitrogen) nylon membranes. The RNA gel blots were hybridized with an $\alpha-{ }^{32} \mathrm{P}$-labeled OSIAA9 sequence-specific probe at $65{ }^{\circ} \mathrm{C}$ overnight. Blots were washed with a Hybond washing buffer, and the signal was detected by radiography. Real-time PCR methods are described in Song et al. (2009b); they used specific primers listed in Table 1 Suppl.

Yeast two-hybrid analysis: Full-length $O_{S I A A}$, OSIAA1, and $O S I A A 4$ were inserted into yeast expression vectors PAD502 and PDBLeu (Invitrogen, Carlsbad, CA, USA). Yeast (Saccharomyces cerevisiae L.) strain MAV502 was used to determine the interaction affinity. A colony-lift filter assay ( $\beta$-galactosidase assay) was performed as described by the manufacturer (Invitrogen), and the results confirmed by growing the transformants on synthetic complete medium without leucine, tryptophan, and histidine (SC-3) and containing $10 \mathrm{mM} \mathrm{3-amino-triazole.}$ Primers used are listed in Table 2 Suppl.

$\boldsymbol{\beta}$-Glucuronidase (GUS) staining: Histochemical GUS analysis was performed as described by Song et al. (2009a). Transgenic plant samples were incubated with a

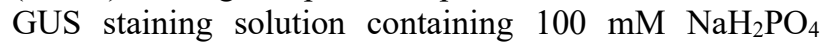
buffer (pH 7.0), $0.5 \%(\mathrm{~m} / \mathrm{v})$ Triton $X-100,0.5 \mathrm{mg} \mathrm{cm}^{-3}$ 5-bromo-4-chloro-3-indolyl- $\beta$-D-glucuronic acid, and $20 \%(\mathrm{v} / \mathrm{v})$ methanol. After staining, the tissue samples were mounted on slides and observed and photographed under a microscope. Seedlings, roots, leaves, and flower organs were incubated in a solution of $1 \mathrm{mM}$ 5-bromo-4chloro-3-indolyl glucuronide and in a $50 \mathrm{mM}$ sodium phosphate buffer (pH 7.0) at $37^{\circ} \mathrm{C}$ for $13-20 \mathrm{~min}$.

Genotyping T-DNA mutant of OsIAA9: A T-DNA mutant of OsIAA9 was obtained from the Rice Mutant database (http://RMD.ncpgr.cn). A PCR was conducted to identify the genotype of the progeny from a T-DNA insertion line for the OSIAA9 gene using three primers: a forward primer ( $5^{\prime}$-CGATCGATCACAACTACTAG-3'), reverse primer ( $5^{\prime}$-GAGATGTGCCCAACTACAGT-3'), and T-DNA-specific left border primer (NTBL5, 5'-AATCCAGATCCCCCGAATTA-3'). 


\section{Results}

In previous analysis of OSIAA gene expression, I noticed that OsIAA9 (AK073365, LOC_Os02g56120) is highly and rapidly induced by auxin and is specifically expressed in some tissues (Song et al. 2009a). Therefore, in this study, I was interested in identifying the function of OSIAA9. Based on phylogenetic analysis, OsIAA9 and OsIAA20 form one subclade (Song et al. 2013). OsIAA9 contains all the domains conserved by Aux/IAA proteins including domain I, represented as LRLGLT; domain II, represented as VVGWPPV; and domains III and IV (Jain et al. 2006). Domains III and IV of Aux/IAA can form homo- or hetero-dimers with other Aux/IAA proteins. As
OsIAA9 is apparently a canonical Aux/IAA protein that harbors domains III and IV, I believed it can form homoor hetero-dimers. To test this hypothesis, full-length OsIAA9, OsIAA1, and OsIAA4 were cloned into PAD502 and PDBLeu. Yeast colony filter assays (Fig. $1 A$ ) and growth of transformants (Fig. $1 B$ ) demonstrated that OsIAA9 can form a homodimer or heterodimer with OsIAA1 (a canonical Aux/IAA protein) and OsIAA4 (a dominant mutation-type domain II Aux/IAA protein). Similar results were reported in identifying the function of OsIAA1 (Song et al. 2009b). These observations indicate that OsIAA9 was a canonical $A u x / I A A$ gene.

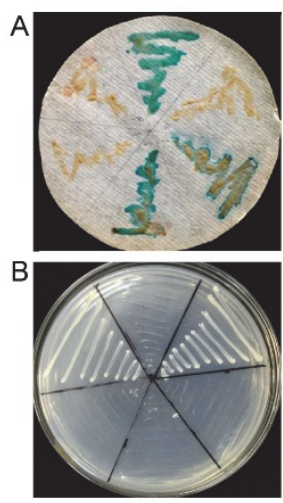

OsIAA1-OsIAA9
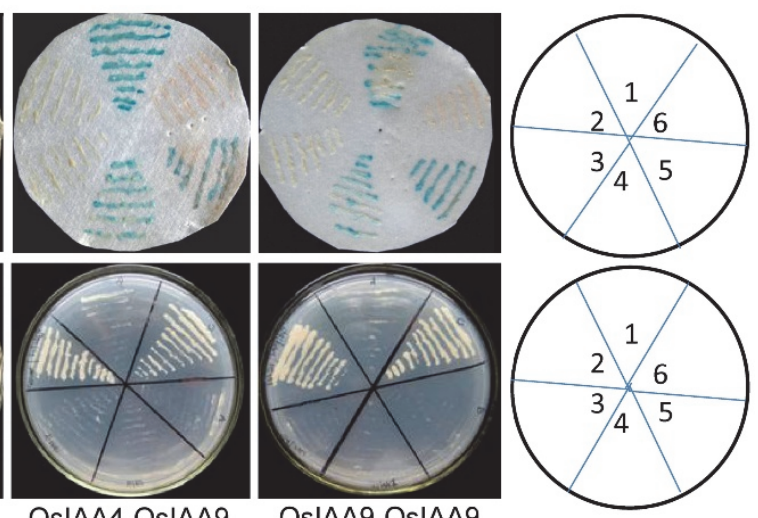

Fig. 1. $A$ - Yeast two-hybrid assay of OsIAA1-OsIAA9, OsIAA4-OsIAA9, and OsIAA9-OsIAA9 in $\beta$-galactosidase colony filter assay (yeast transformants were grown on YPAD medium and colony filter-lift assays were perfomed). 1 - pBD-OsIAAx $(\mathrm{X}=1,4$, and 9 , as indicted in the figure) + pAD-OsIAA9; 2 - pBD-OsIAAx (X = 1, 4, and 9, as indicated); 3 - pAD-OsIAA9; 4 - positive control; 5 - positive controls (positive 4 is a little bit stronger than 5); 6 - negative control. $B$ - Growth of yeast transformants on synthetic complete medium without leucine, tryptophan, and histidine containing $10 \mathrm{mM} \mathrm{3-aminotriazole.} 1$ - negative control; 2 - positive control; 3 - pAD-OsIAA9; 4 - pBD-OsIAAx $(X=1$, 4, and 9); 5 - negative control; 6 - pBD-OsIAAx $(X=1,4$, and 9) + pAD-OsIAA9. ( 1 and 5 are the same transformants). (X stand for different OsIAA genes, OsIAA1 on left, OsIAA4 on middle and OsIAA9 on right)

Tissue and organ expression patterns from collection of rice expression profiles CREB (http://crep.ncpgr.cn; Wang et al. 2010) showed that more OSIAA9 transcript accumulates in the callus, endosperm, radicle, and seed after imbibition (Song et al. 2009a). To further investigate the spatial and developmental regulation of OSIAA9, I cloned the OSIAA9 promoter to drive GUS expression and introduced the construct into rice for histochemical analysis. Around 10 independent T2 transgenic lines containing the OSIAA9 promoter constructs were analyzed throughout the life cycle of rice. The OSIAA9 was expressed in roots and seedling shoots (Fig. 2) but not in any other tissues or organs (data not shown). Detailed histochemical analyses of the GUS activity of $\mathrm{P}_{\text {OSIAA9: GUS }}$ lines show that OSIAA9 was expressed in the coleoptiles and roots at the seedling stage (Fig. $2 A, B$ ), including the root tip (Fig. $2 C$, lateral root stele (Fig. $2 D$ ), and lateral roots (Fig. 2E,F) suggesting a role of OSIAA9 in root development, especially of lateral roots. The OSIAA9 promoter activity was visible during early lateral root formation at the sites of primordia initiation and was very high in the vasculature of the emerging lateral roots (Fig. 2E,F).

To find putative $c i$-elements of OSIAA9, I analyzed the promoter of 2000-bp regions upstream from ATG using the PLACE 30.0 database (Higo et al. 1999). The search results suggest that the sequence of this fragment contained numerous DNA elements predicted to be responsive to auxin, drought, abscisic acid (ABA), salicylic acid (SA), and high salinity. The DNA elements included three copies of TGTCTC (AuxREs for ARF binding); two copies of ACGTG (a drought-inducible ABRE-like element); a copy of CACGTG (ABAinducible); two copies of TGACG (IAA/SA-inducible); a copy of TTGAC (SA-inducible); several copies of GNGGTG, GTGGNG, and GAAAAA (salt-inducible); and two copies of TGACG and GCCGCC (JA-related element) (data not shown). These predictions suggest that the functions of OSIAA9 might be associated with various phytohormone signals and abiotic stresses. To further explore the possible involvement of OsIAA9 in the regulation of hormone- and abiotic stress-related genes, 
the expressions of OSIAA9 in response to exogenous hormones (IAA, 2,4-D, JA, SA, gibberellic acid, ABA, and kinetin) and abiotic stresses (drought and salt) were examined by real-time PCR. The OsIAA9 was strongly induced by IAA (7-fold up-regulated), 2,4-D (10-fold upregulated), JA (12-fold up-regulated), SA (5-fold up- regulated), and salinity (10-fold up-regulated) and slightly stimulated by drought (3-fold up-regulated), but no significant changes were detected following treatment with ABA, kinetin, or gibberellic acid (Fig. 3). These results suggest that $O S I A A 9$ had multiple functions in rice.
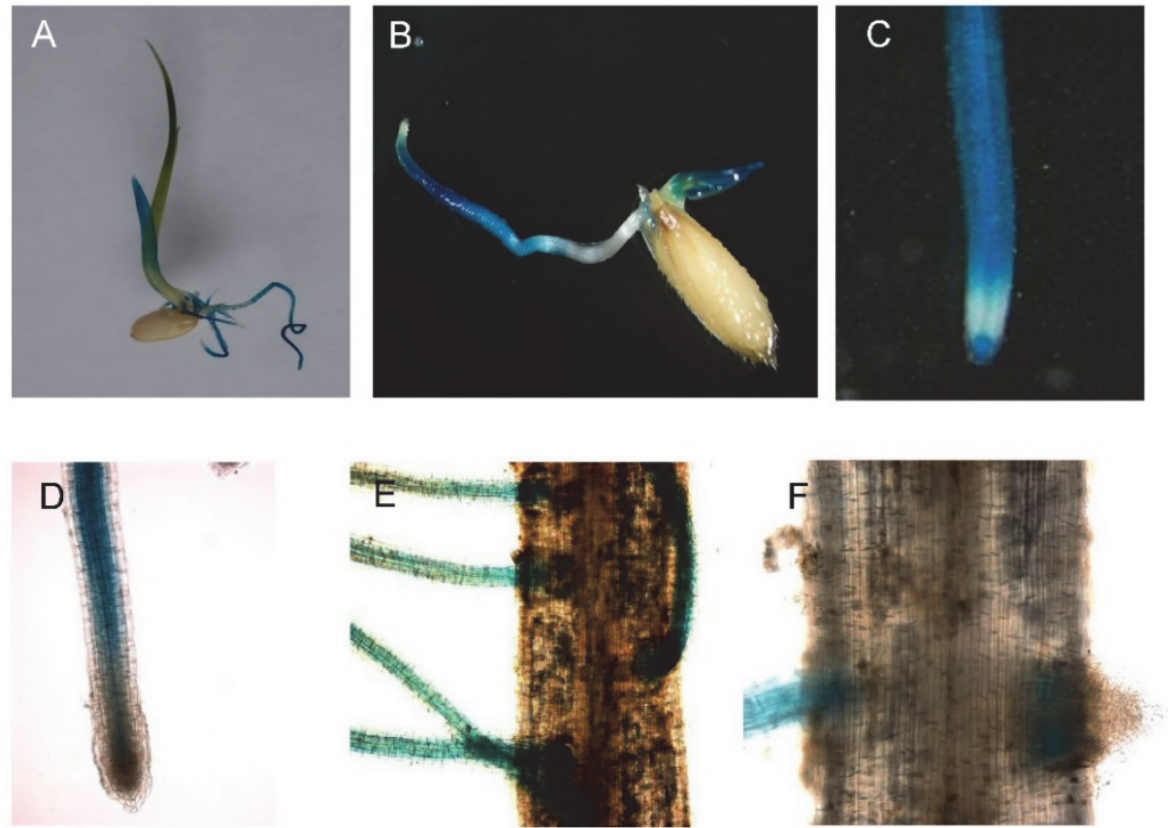

Fig. 2. An expression pattern of OSIAA9 as indicated by the $\beta$-glucuronidase (GUS) reporter gene driven by the OsIAA9 promoter. The GUS expression in seedling $(A, B)$, the seminal root tip and root stele (the elongation zone) $(C)$, the root tip of lateral root $(D)$, lateral roots $(E)$, at the base of lateral $\operatorname{root}(F)$.

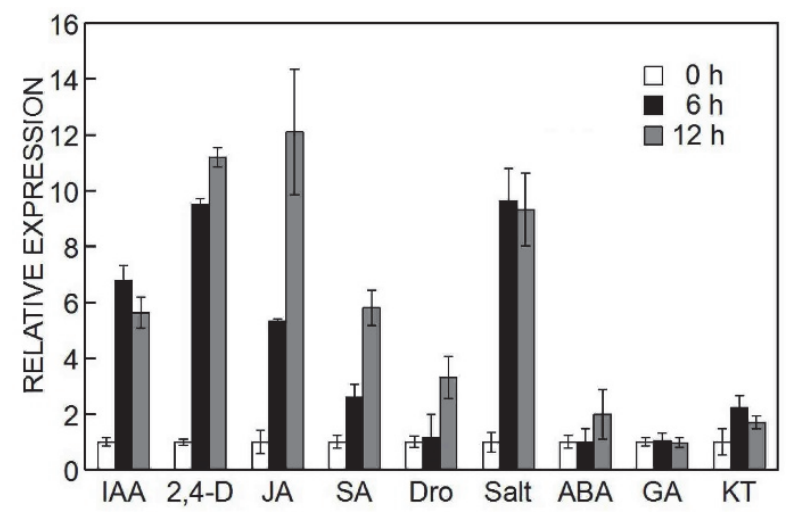

Fig. 3. Relative expressions (normalized to time point 0 in each treatment) of OsIAA9 in response to hormones and abiotic stresses (see materials and methods for details and abbreviations) as determined by real-time PCR.

To further test the function of OsIAA9, I cloned fulllength $O S I A A 9$ driven by the maize ubiquitin promoter and transformed it into rice (Fig. 4A). Leaves of all 22 independent lines of transgenic plants were collected to detect the expression of OsIAA9. Analyses of the RNA-blot indicate 13 overexpression lines relative to the wild type (Fig. 4B). The OsIAA1-overexpressing plants exhibited semi-dwarfism and increased leaf angles (Song et al. 2009b) and OsIAA4-overexpressing plants exhibited semidwarfism and increased tiller angles (Song et al. 2013). When I observed OSIAA9-overexpressing transgenic plants in the field, no obvious morphological changes in plant height, tiller number, leaf angle, or tiller angle (data not shown) were found.

Auxins play crucial roles in root development including regulation of growth of the main root in 
Arabidopsis and the seminal root in rice. Because OsIAA9 responds rapidly and highly to auxins, all OsIAA9 protein has the typical Aux/IAA conserved domains including domain I, II, III and IV. I next focused on the root morphology of OsIAA9-overexpressing plants. Root elongation assays are a typical way to investigate the responses of genes to hormone application. To investigate the function of OSIAA9 in the auxin signalling pathway, I tested the responsiveness of OSIAA9-overexpressing transgenic plants to application of 2,4-D, a synthetic auxin (Song et al. 2014). Five independent positive lines (O2, $\mathrm{O} 11, \mathrm{O} 12, \mathrm{O} 17$, and O19) and one non-overexpressing line $(\mathrm{O} 21)$ were chosen for a root elongation essay. I first tested the responsiveness of OSIAA9-overexpressing plants in an MS medium containing $0.1 \mu \mathrm{M}$ 2,4-D. The OsIAA9overexpressing plants grew better than WT plants in medium containing $0.1 \mu \mathrm{M}$ 2,4-D (Fig. $4 D-I$ ), whereas no differences were observed for the wild type or non- overexpressing line $\mathrm{O} 21$ (Fig. 4C) suggesting that overexpressing OSIAA9 in rice led to resistance to exogenous auxin. Then, dose responsiveness of OSIAA9overexpressing plants to $2,4-\mathrm{D}$ was tested. The OsIAA9overexpressing plants were grown in an MS medium containing different concentrations of 2,4-D, and data were collected after two weeks of growth. The roots of transgenic lines were significantly longer than the roots of the wild type (Fig. 4J), further confirming OsIAA9overexpressing plants were less sensitive to the inhibitory effect of auxin than the wild type. Finally, the responsiveness of OSIAA9-overexpressing plants to the natural auxin IAA were tested. Transgenic plants exhibited resistance to IAA compared to the wild type (data not shown).

To characterize the root phenotype of OSIAA9overexpressing plants under normal growth conditions, germinated seeds of OSIAA9-overexpressing plants and
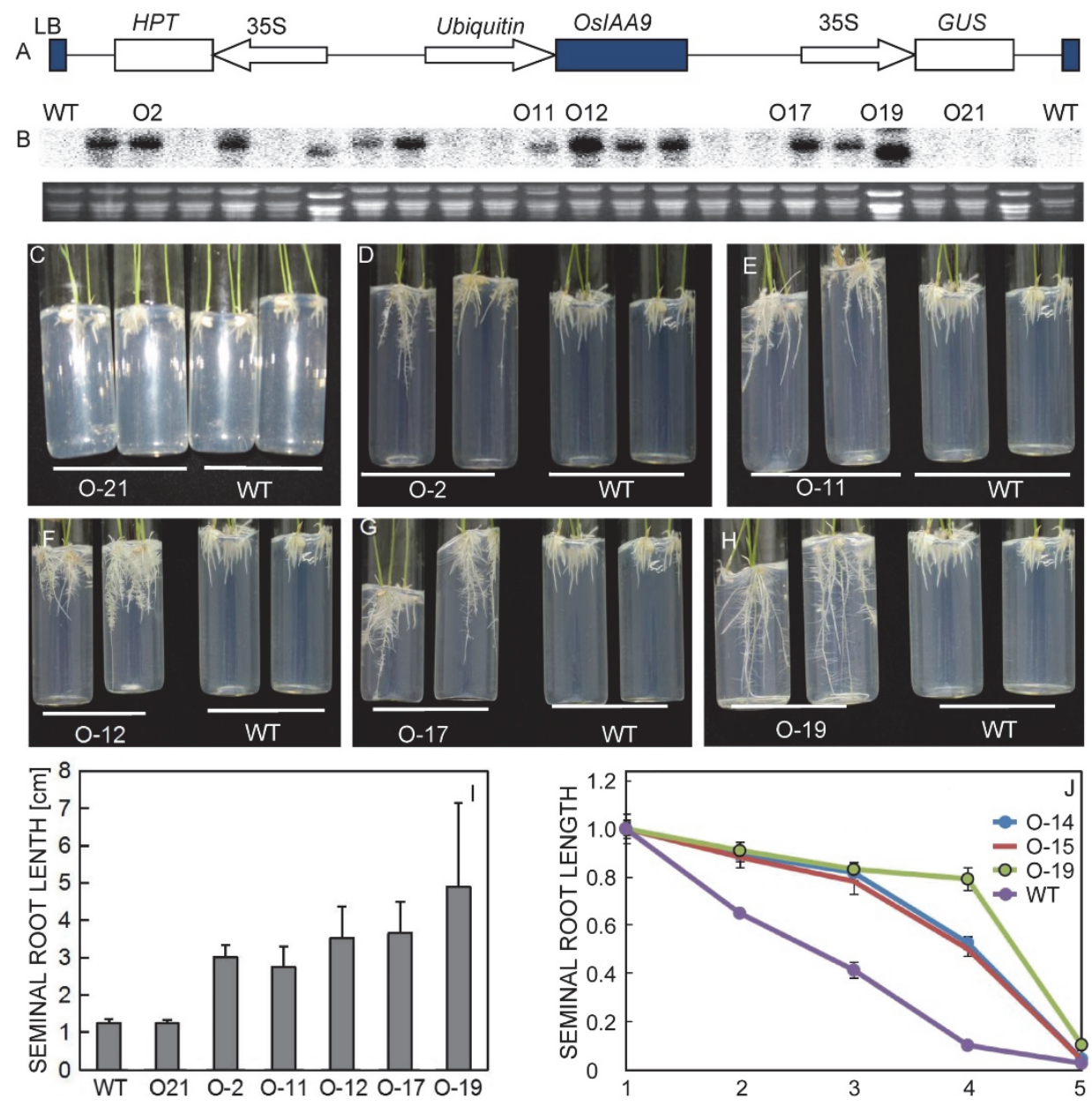

Fig. 4. Root elongation assay of OSIAA9-overexpressing plants treated with 2,4-D. The overexpression construct for rice transformation $(A)$. The RNA gel-blot analysis of transgenic and wild-type (WT) plants $(B)$. Transgenic overexpressing plants O-2 $(D)$, O-11 $(E)$, O-12 $(F)$, O-17 $(G)$, and O-19 $(H)$, non-overexpressing transgenic plant O-21, and WT plants were grown in an Murashige and Skoog medium with $0.1 \mu \mathrm{M}$ 2,4-D. Quantification of seminal root length in $D-F(I)$. The germinated seeds of OsIAA9overexpressing (O-12, O-17, and O-19) and WT plants were transferred to MS medium containing 0 (1), 0.01 (2), 0.05 (3), 0.1 (4), and 0.5 (5) $\mu \mathrm{M} 2,4-\mathrm{D}$ and root lengths was measured after two weeks $(J)$. Error bars indicate SE, $n=20$. 
wild-type plants were transferred to an MS medium to observe differences in their growth (Fig. 5A). One of the most striking characteristics of ectopic overexpression of OsIAA9 was a decrease in the number of crown roots (Fig. 5B,C). In two-week-old wild-type seedlings grown in tubes, the crown root number was about eight per plant (Fig. 5D). However, OSIAA9-overexpressing transgenic plants had only about four crown roots per plant (Fig. 5D). The second strong feature of the phenotype was a decrease in the number of lateral roots (Fig. $5 E, F$ ). The lateral root density was about 18 roots $\mathrm{cm}^{-1}$ in the wild type but about

10 roots $\mathrm{cm}^{-1}$ in $O s I A A 9$-overexpressing plants (Fig. $5 G$ ). To determine whether exogenous auxin affects this reduction in number of crown roots in OSIAA9overexpressing plants, the wild-type and OSIAA9overexpressing plants were grown in an MS medium both with and without 2,4-D. Taken together, these observations suggest that ectopic overexpression of $O S I A A 9$ resulted in a decreased crown root number, caused defective lateral root formation, and impaired auxin sensitivity in roots.
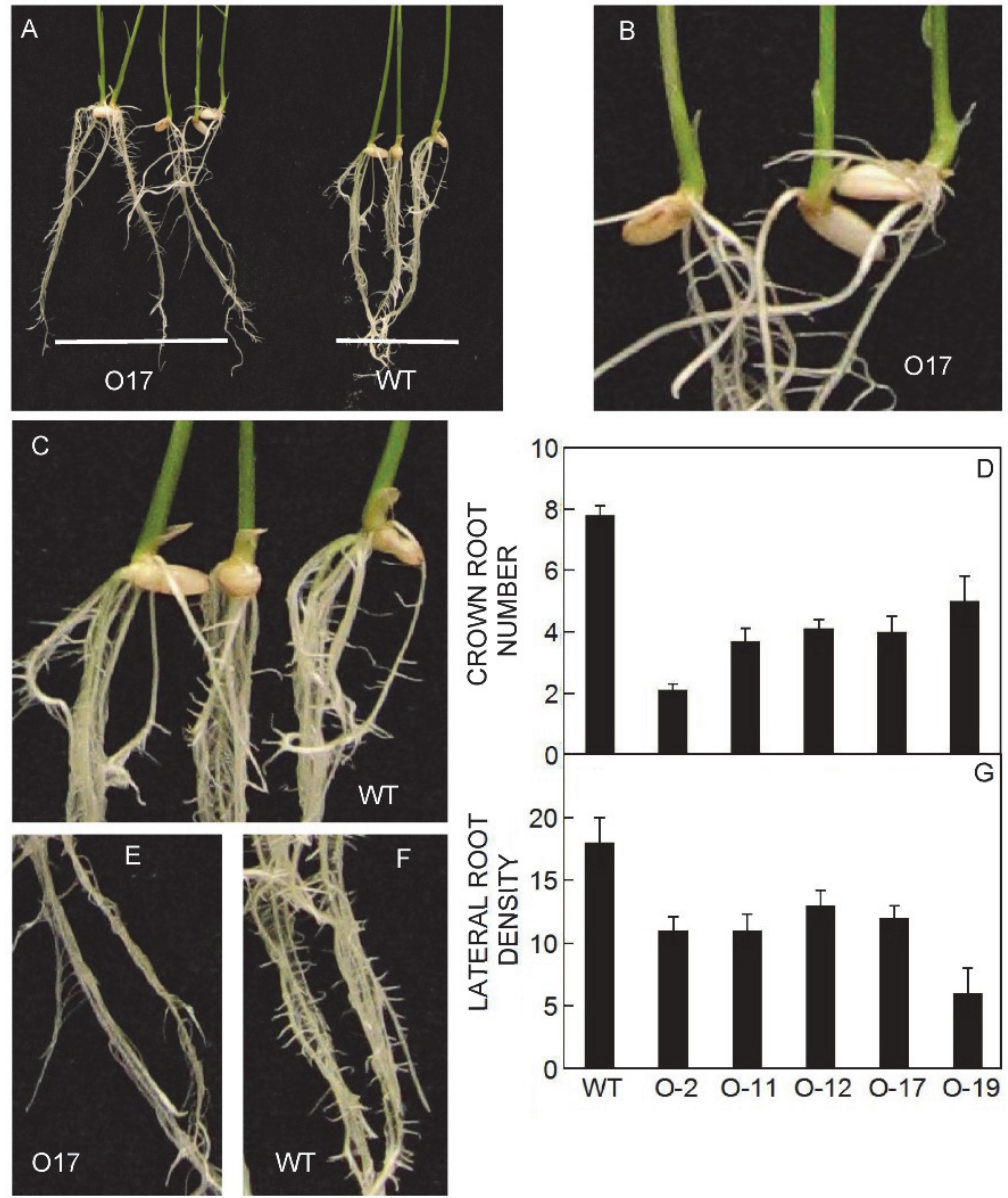

Fig. 5. Root phenotypes of OSIAA9-overexpressing plants. Appearance of OsIAA9-overexpressing plants and wild-type plants under normal growth conditions $(A)$. The crown root number of OsIAA9-overexpressing plants $(B)$ and wild-type plants under normal growth conditions $(C)$. Quantification of the number of crown roots in OsIAA9-overexpressing and wild-type plants $(D)$. Root morphology of OsIAA9-overexpressing plants $(E)$ and wild-type plants under normal growth conditions $(F)$. Lateral root density of OSIAA9overexpressing and wild-type plants $(G)$. Error bars indicate SE, $n=20$.

To further characterize the function of OSIAA9, I crossed a mutant of OSIAA9 with T-DNA inserted in the first intron (Fig. 6A) to the wild type for homozygosity testing. Out of 12 T1 generation plants, 3 were homozygous (M1, M6, and M12), 5 were heterozygous (M4, M5, M7, M8, and M9), and 4 corresponded to the wild type (M2, M3, M10, and M11) (Fig. 6B). Seminal root length was checked under normal growth conditions or after treatment with auxin; no significant difference was observed between homozygous (M12) and wild-type plants (M10) (Fig. 6C) suggesting functional redundancy of Aux/IAA proteins.

Auxin/IAA proteins possess a potent transcriptional co-repression activity through domain I, which contains an EAR [ethylene response factor (ERF)-associated amphiphilic repression (EAR), defined by the consensus sequence patterns of either LxLxL or DLNxxP] motif for interaction with the co-repressor protein TOPLESS 
(Tiwari et al. 2004). Protein sequence analysis showed that OsIAA9 harbors domain I. To determine whether OsIAA9 functions as a co-repressor of transcriptional regulation of auxin responses, as do other Aux/IAA proteins, I examined the effects of OSIAA9 overexpression on the induction of early auxin responsive genes, including OSIAA1, OSIAA4, OSIAA9, and OSIAA20, in the whole plant. The induction of three canonical $A u x / I A A$ genes, OsIAA1, OsIAA9 itself, and OsIAA20, was suppressed by exogenous auxin application in OSIAA9-overexpressing plants, whereas the induction of one non-canonical $A u x / I A A$ gene, OsIAA4, was not significantly influenced by exogenous auxin (Fig. 2 Suppl.) suggesting that ectopic overexpression of OSIAA9 repressed auxin-induced gene expression.

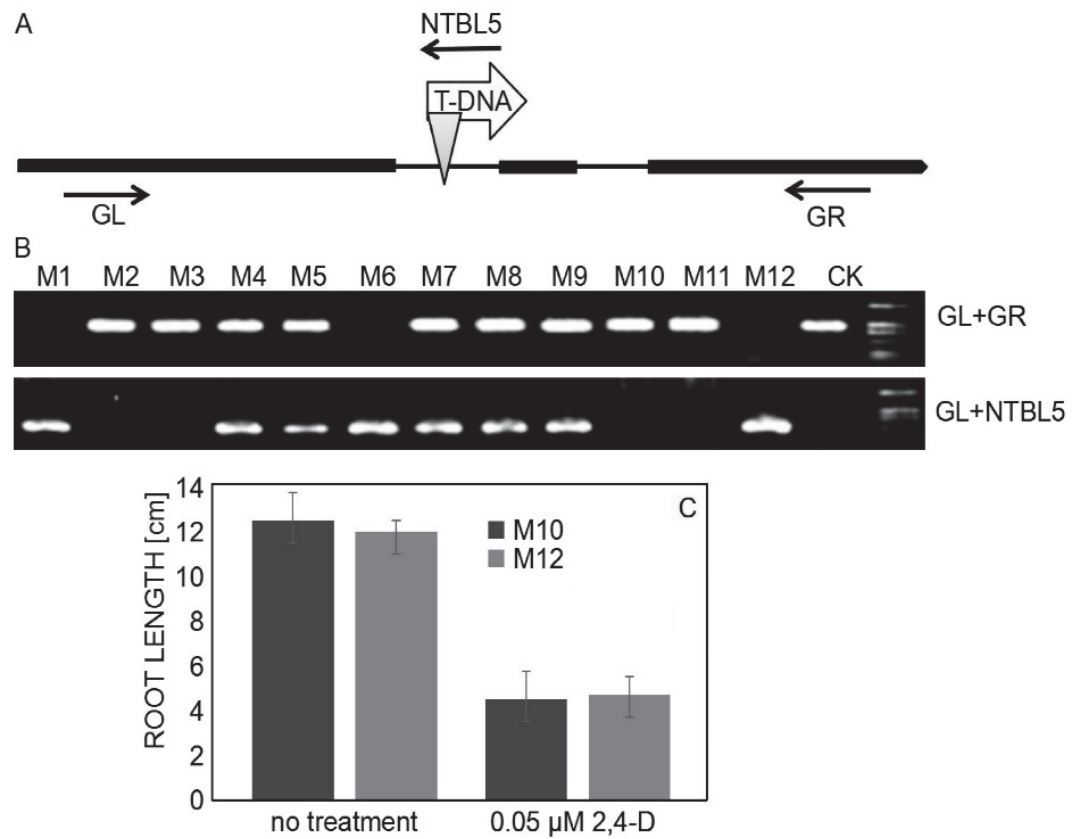

Fig. 6. A - Characterization of osiaa9 mutants. The T-DNA insertion site in the OsIAA9 gene. Boxes and lines (not drawn to scale) represent exons and introns, respectively. $B$ - The insertion site and genotype of the mutant as confirmed by PCR using a pair of genespecific primers (GL+GR) and a T-DNA border primer (NTBL5). $C$ - Seminal root length with and without 2,4-D treatment. Homozygous mutant (M12) and wild-type (M10) seeds were germinated on an Murashige and Skoog medium and transferred to an MS medium containing 0 or $0.05 \mu \mathrm{M} 2,4-\mathrm{D}$. Means $\pm \mathrm{SE}, n=20$.

To further check the regulation of auxin signaling by OSIAA9, the expression of OSIAA9 in OSIAA1- and OSIAA4-overexpressing transgenic plants was tested. The induction of OSIAA9 in OSIAA1-overexpressing plants was

\section{Discussion}

Auxin is an important phytohormone that plays a crucial role in plant growth and development, acting either alone or interplaying with other phytohormones. One of the best known functions of auxin is its regulation of root development. It facilitates root growth and elongation (Peret et al. 2009, Peer et al. 2011) and is required for the initial cell division during development of lateral root primordia (De Smet et al. 2006, 2007). In this study, I focused on identifying a canonical $A u x / I A A$ gene, OsIAA9, in rice; promoter sequence analysis of OSIAA9 identified several cis-elements related to auxin, SA, drought, and salinity. Real-time PCR shows that the expression of OsIAA9 was highly induced by IAA, 2,4-D, SA, and JA implying multiple functions of OSIAA9 in hormone significantly suppressed (Fig. 3A Suppl.); however, there was no clear change in OSIAA4-overexpressing plants (Fig. $3 B$ Suppl.). Taken together, these results suggest that OSIAA9 was a major auxin negative regulator in rice. mediated responses. The OsIAA9 was also highly induced by salinity and slightly induced by drought but no significant change was found after ABA treatment indicating that OSIAA9 may mediate plant abiotic stress responses independent of the ABA pathway. The $\beta$-glucuronidase staining shows OSIAA9 mainly as a root specifically expressed gene. Ectopic overexpressions of OSIAA1 and OSIAA4 in rice cause multiple phenotypic changes including a decreased plant height, increased leaf/tiller angle, and insensitivity to auxin in roots (Song et al. 2009b, Song and $\mathrm{Xu}$ 2013). Unlike OsIAA1 and OSIAA4, however, ectopic overexpression of OSIAA9 did not cause any visible phenotypic change in aboveground parts, but did reduce inhibition of root elongation in 
response to exogenous auxin. One explanation for the difference between OsIAA9 and OSIAAI/OsIAA4 ectopic overexpression in aboveground parts may be due to the different expression pattern of each gene. The OSIAA1 is highly expressed throughout the entire life cycle (Song et al. 2009b), and OsIAA4 shows a very low expression throughout the entire life cycle (Song and Xu 2013), whereas $O S I A A 9$ was specifically expressed in seedling roots and shoots.

Interactions between phytohormones play important roles in the regulation of plant growth and development. There is an increasing evidence of extensive interaction between phytohormones also in the adaptation to external stimuli (Wolters and Jurgens 2009). These interactions are in some cases governed by shared components of signal transduction pathways, but in other cases reflect the modulation that a given hormone exerts on the synthesis or action of another (Hoffmann et al. 2011). The OSIAA9 is highly induced by auxin and JA implying that OSIAA9 may act through $O_{S A R F \mathrm{~s}}$ to influence crosstalk between auxin and JA. Further work is need to determine the phenotypic characterization of OSIAA9 in JA response and to identify how $O S A R F$ mediates the interplay between JA and auxin responses in rice.

Salinity is one of the most common environmental stresses with detrimental effects on plant growth and development (Munns and Tester 2008). When plants are exposed to stresses, they often respond with a partial or complete arrest of growth. This response is considered an adaptive strategy for survival enabling plants to reallocate multiple resources towards combating stress (Xiong and Zhu 2001). Plants use two different strategies to deal with a high soil salinity. One strategy is activation of pathways that allow the plant to export or compartmentalize salt. Relying on their phenotypic plasticity, the second strategy is the adjustment of their root system architecture and the direction of root growth to avoid locally high salt concentrations (Galvan-Ampudia and Testerink 2011). Modulation of root system architecture by salt stress involves differential regulation of auxin distribution in the root, which determines both direction of growth and development of lateral roots (Galvan-Ampudia and Testerink 2011). In this study, I observed that OSIAA9 was significantly accumulated under salt treatment and slightly under drought stress. The OSIAA9 may function at the intersection between auxin and abiotic stress signalling. Furthermore, OSIAA9-overexpressing Arabidopsis shows an enhanced tolerance to osmotic stress and salt stress (http://www.saltscience.or.jp/g_summary/2009/0905E.pdf), and OsIAA9 is mainly a root-expressed gene with an ectopic overexpression of OSIAA9 in rice causing an abnormal root phenotype. All these results imply that OSIAA9 may be involved in adjustment of root system architecture and the direction of root growth to avoid locally high salt concentrations.

Auxin signalling transduction depends on the rapid degradation of Aux/IAA proteins. The Aux/IAA proteins function as negative feedback regulators to modulate the exact regulation of auxin related physiological process. The high auxin responsiveness of OSIAA9 shows that it may be an important auxin negative feedback regulator, and the inhibition of expression of OSIAA9 in OSIAA1overexpressing plants, and the suppression of several auxin response OSIAAs in OSIAA9-overexpressing plants suggest that OSIAA9 is an important auxin negative feedback regulator in rice.

\section{References}

De Smet, I., Tetsumura, T., De Rybel, B., Frey, N.F., Laplaze, L., Casimiro, I., Swarup, R., Naudts, M., Vanneste, S., Audenaert, D., Inze, D., Bennett, M.J., Beeckman, T.: Auxindependent regulation of lateral root positioning in the basal meristem of Arabidopsis. - Development 134: 681-690, 2007.

De Smet, I., Vanneste, S., Inze, D., Beeckman, T.: Lateral root initiation or the birth of a new meristem. - Plant mol. Biol. 60: 871-887, 2006.

Galvan-Ampudia, C.S., Testerink, C.: Salt stress signals shape the plant root. - Curr. Opin. Plant Biol. 14: 296-302, 2011.

Higo, K., Ugawa, Y., Iwamoto, M., Korenaga, T.: Plant cis-acting regulatory DNA elements (PLACE) database: 1999. Nucleic Acids Res. 27: 297-300,1999.

Hoffmann, M., Hentrich, M., Pollmann, S.: Auxin-oxylipin crosstalk: relationship of antagonists. - J. integr. Plant Biol. 53: 429-445, 2011.

Jain, M., Kaur, N., Garg, R., Thakur, J.K., Tyagi, A.K., Khurana, J.P.: Structure and expression analysis of early auxinresponsive Aux/IAA gene family in rice (Oryza sativa). Funct. integr. Genomics 6: 47-59, 2006.

Jenik, P.D., Barton, M.K.: Surge and destroy: the role of auxin in

plant embryogenesis. - Development 132: 3577-3585, 2005.

Kitomi, Y., Inahashi, H., Takehisa, H., Sato, Y., Inukai, Y.: OsIAA13-mediated auxin signaling is involved in lateral root initiation in rice. - Plant Sci. 190: 116-122, 2012.

Luo, S., Li, Q., Liu, S., Pinas, N.M., Tian, H., Wang, S.: Constitutive expression of OsIAA9 affects starch granules accumulation and root gravitropic response in Arabidopsis. Front. Plant Sci. 6: 1156, 2015.

Leyser, O.: The fall and rise of apical dominance. - Curr. Opin. Genet. Dev. 15: 468-471, 2005.

Muday, G.K., DeLong, A.: Polar auxin transport: controlling where and how much. - Trends Plant Sci. 6: 535-542, 2001.

Munns, R., Tester, M.: Mechanisms of salinity tolerance. - Annu. Rev. Plant Biol. 59: 651-681, 2008

Ni, J., Wang, G.H., Zhu, Z.X., Zhang, H.H., Wu, Y.R., Wu, P.: OsIAA23-mediated auxin signaling defines postembryonic maintenance of QC in rice. - Plant J. 68: 433-442, 2011.

Peer, W.A., Blakeslee, J.J., Yang, H.B., Murphy, A.S.: Seven things we think we know about auxin transport. - Mol. Plant. 4: 487-504, 2011.

Peret, B., De Rybel, B., Casimiro, I., Benkova, E., Swarup, R., Laplaze, L., Beeckman, T., Bennett, M.J.: Arabidopsis lateral 
root development: an emerging story. - Trends Plant Sci. 14: 399-408, 2009.

Rebouillat, A., Dievart, J., Verdeil, J., Escoute, J., Giese, G., Breitler, J.C., Gantet, P., Espeout, S., Guiderdoni, E., Perin, C.: Molecular genetics of rice root development. - Rice 2: 1534, 2009.

Song, Y.L.: Insight into the mode of action of 2,4dichlorophenoxyacetic acid (2,4-D) as an herbicide. - J. integr. Plant Biol. 56:106-13, 2014.

Song, Y.L., Wang, L., Xiong, L.Z.: Comprehensive expression profiling analysis of OSIAA gene family in developmental processes and in response to phytohormone and stress treatments. - Planta 229: 577-591, 2009a.

Song, Y.L., $\mathrm{Xu}, \quad$ Z.F.: Ectopic overexpression of an AUXIN/INDOLE-3-ACETIC ACID (Aux/IAA) gene OSIAA4 in rice induces morphological changes and reduces responsiveness to auxin. - Int. J. mol. Sci. 14: 13645-13656, 2013.

Song, Y.L., You, J., Xiong, L.Z.: Characterization of OsIAA1 gene, a member of rice $A u x / I A A$ family involved in auxin and brassinosteroid hormone responses and plant morphogenesis. - Plant mol. Biol. 70: 297-309, 2009b.

Tiwari, S.B., Hagen, G., Guilfoyle, T.J.: Aux/IAA proteins contain a potent transcriptional repression domain. - Plant Cell 16: 533-543, 2004.

Wang, L., Xie, W.B., Chen, Y., Tang, W.J., Yang, J.Y., Ye, R.J., Liu, L., Lin, Y.J., Xu, C.G., Xiao, J.H., Zhang, Q.F.: A dynamic gene expression atlas covering the entire life cycle of rice. - Plant J. 61: 752-766, 2010.

Wolters, H., Jurgens, G.: Survival of the flexible: hormonal growth control and adaptation in plant development. - Nat. Rev. Genet. 10: 305-317, 2009.

Xiong, L.M., Zhu, J.K.: Abiotic stress signal transduction in plants: molecular and genetic perspectives. - Physiol. Plant. 112: 152-166, 2001.

Zhu, Z.X., Liu, Y., Liu, S.J., Mao, C.Z., Wu, Y.R., Wu, P.: A gainof-function mutation in OSIAA11 affects lateral root development in rice. - Mol. Plant. 5: 154-161, 2012. 